\title{
Optimization of Cryopreservation Process Using Response Surface Methodology for Chlorella saccharophila and Chlorella zofingiensis
}

\author{
Zeliha Demirel $^{1 *}$, Esra Imamoglu ${ }^{1}$, Irem Deniz $^{2}$, Meltem Conk Dalay $^{1}$ \\ ${ }^{1}$ Ege University, Faculty of Engineering, Department of Bioengineering, Izmir, Turkey \\ ${ }^{2}$ Manisa Celal Bayar University, Faculty of Engineering, Department of Bioengineering, Manisa, Turkey. \\ "zelihademirel@gmail.com
}

Received: 23 May 2018

Accepted: 10 December 2018

DOI: $10.18466 /$ cbayarfbe. 426444

\begin{abstract}
The preservation of microalgae in a stable state is a fundamental requirement in pharmaceutical, agricultural, environmental sciences and different industries. Cryopreservation is widely stabilized for achieving long-term storage and has been applied to an increasingly diverse range of microalgae and cell cultures. The continuous storage of actively growing microalgae strains by routine serial subculture is relatively time-consuming and this technique has possible contamination risks. In this study, the optimization of cryopreservation process was carried out for two different Chlorella strains using response surface methodology (RSM) with three factors (cryoprotectant concentration, incubation time and cryopreservation time) including 19 runs. The optimal cell viability of $C$. zofingiensis was found at the dimethyl sulfoxide (DMSO) concentration of $12.89 \%$ at the incubation time of 8.14 min and with the cryopreservation time of 93.45 day, while $C$. saccharophila was found at the DMSO concentration of 12.86 $\%$ at the incubation time of $7.99 \mathrm{~min}$ and at cryopreservation time of 95.17 day.
\end{abstract}

Keywords: Cryopreservation; Chlorella saccharophila; Chlorella zofingiensis; response surface methodology

\section{Introduction}

Microalgae are foremost biomass sources for health foods, feed additives, cosmetics and biodiesel production due to their benefits, such as not competing for arable land with crops, high influent in capturing sunlight, and decreasing $\mathrm{CO}_{2}$ emissions compared to terrestrial flora [1].

Long-term stability of microalgae culture collections in serial sub-culturing using liquid or solid media cannot be preserved because there are labor intensive, costly and a risk of contamination and genetic alterations [2] Therefore cryopreservation, the preservation of cellular viability at low sub-zero temperature, provides a crucial option for conserving a microalga for weeks or even years [3]. Cryopreserved cells are protected from any genetic changes and required minimum maintenance and labor, believing in stored during suitable conditions; contamination risk of other microorganisms should decreased [4]. Although, the most important problem about cryopreservation process of microalgae is formed severe osmotic stress and/or ice crystal damage throughout the process both freezing and thawing [5]. It is reported that metabolic changes lead to cryopreservation can figure out intracellular free radicals by cryoprotectant exposure and cooling process [6]. Thus, microalgae cell viability after cryopreserved was analyzed by response surface methodology (RSM) used to evaluate optimal conditions for cryoprotectant concentration, incubation time with cryoprotectant at room temperature and cryopreservation time. For the optimization of algal cell viability, it is necessary to optimize these factors with an optimization method. In that, RSM is empirical statistical modeling techniques on the multivariate non-linear model-based that is able to work out interactions among all parameters [7].

In this work, the information was given on changes in viability of cryopreserved two different Chlorella harvested from late exponential of culture. In this study, optimization of cryopreservation conditions were conducted with 3 different parameters of cryoprotectant concentration $(0-25 \%)$, pretreatment $(1-15 \mathrm{~min})$ the duration of cryopreservation (7-180 day) for $C$. saccharophila and $C$. zofingiensis by central composite design (CCD) using response surface methodology (RSM) to statistically utilize findings.

\section{Material and methods \\ 2.1 Cultivation of algae}

Two freshwater strains, Chlorella saccharophila (Krüger) Migula (EGEMACC 13) and C. zofingiensis Dönz (EGEMACC 20) were supplied from Ege Microalgae Culture Collection (EGEMACChttp://www.egemacc.com/). The microalgae cells were monoalgal and growth in $100 \mathrm{~mL}$ Bold Basal Medium (BBM) [8] of $250 \mathrm{~mL}$ flask, at $22 \pm 2{ }^{\circ} \mathrm{C}$ under $100 \mu \mathrm{mol}$ 
photons $\mathrm{m}^{-2} \mathrm{~s}^{-1}$ until cells reached to latter part of the logarithmic growth phase. Then, cells harvested with centrifuge, resuspended with fresh BBM and cell concentration were counted with Neubauer hemocytometer, and then adjusted to $10^{7}$ cell $/ \mathrm{mL}$.

\subsection{Evaluation of design and data analysis}

The optimization of cryopreservation process was identified by Response Surface Methodology (RSM) based on Central Composite Design (CCD) by the aid of software package Design Expert (version 7.0.0; StatEase Inc., Minneapolis, MN, USA). Dimethyl sulfoxide (DMSO) was used as a cryoprotectant and prepared percent concentrations (Table 1) in the study. CCD analysis was used to determine the impact on three independent parameters (DMSO percent concentration, incubation time in room temperature, cryopreservation time) in 19 runs (Table 2).

Table 1. Calculations for preparing percent concentration.

\begin{tabular}{|c|c|c|}
\hline Concentration (\%) & Cryoprotectant DMSO $(\boldsymbol{\mu L})$ & Cell suspension $(\boldsymbol{\mu} \mathbf{L})$ \\
\hline 0 & - & 1500 \\
\hline 5 & 75 & 1425 \\
\hline 13 & 195 & 1305 \\
\hline 20 & 300 & 1200 \\
\hline 25 & 375 & 1125 \\
\hline
\end{tabular}

Table 2. The experimental response surface design matrix with the actual model-based

\begin{tabular}{|c|c|c|c|}
\hline Treatment & DMSO \% & Incubation time (min) & Cryopreservation time (day) \\
\hline 1 & 13 & 8 & 94 \\
\hline 2 & 20 & 4 & 42 \\
\hline 3 & 20 & 4 & 94 \\
\hline 4 & 13 & 8 & 42 \\
\hline 5 & 5 & 12 & 145 \\
\hline 6 & 5 & 4 & 42 \\
\hline 7 & 5 & 4 & 94 \\
\hline 8 & 13 & 8 & 94 \\
\hline 9 & 13 & 1 & 94 \\
\hline 10 & 13 & 15 & 94 \\
\hline 11 & 13 & 8 & 7 \\
\hline 12 & 13 & 8 & 145 \\
\hline 13 & 5 & 12 & 145 \\
\hline 14 & 20 & 12 & 180 \\
\hline 15 & 13 & 8 & 94 \\
\hline 16 & 0 & 8 & 42 \\
\hline 17 & 20 & 12 & 94 \\
\hline 18 & 25 & 8 & 94 \\
\hline 19 & 13 & 8 & \\
\hline
\end{tabular}

ANOVA was utilized to peruse the statistical significance of regression coefficients via performing the F-test. The most accurate model formed and exhibited in graphical representations with contour plots of factors that represent their relative influence and optimal parameter values. A quadratic polynomial empirical model was in use stated optimum conditions for cryopreserved $C$. saccharophila and $C$. zofingiensis. The second order quadratic polynomial empirical model was used to identify models (2.1):

$Y=\beta_{0}+\sum_{i=1}^{3} \beta_{i} X_{i}+\sum_{i=1}^{3} \beta_{i i} X_{i}^{2}+\sum_{i=1}^{2} \sum_{j=i+1}^{3} \beta_{i j} X_{i} X_{j}$ (2.1)

where $Y$ represents the response $(750 \mathrm{~nm}$ and $665 \mathrm{~nm}$ for C. saccharophila and C. zofingiensis, respectively), $\beta_{0}$ is the interception coefficient; $\beta i$ and $\beta i i$ are the first and second order quadratic design coefficients, respectively; $\beta i j$ is the linear design coefficient for the interaction between factors $i$ and $j$; $\mathrm{X}$ is the factor.

\subsection{Cryopreservation Process}

In this study, the percent concentrations of DMSO are shown in Table 1. DMSO and cell suspension (the concentration of DMSO percentage) were added into cryogenic vials, incubated in room temperature (incubation time) according to the set of experiments (Table 2). After prepared all cryogenic vials, the cells were firstly incubated at $-20^{\circ} \mathrm{C}$ for $30 \mathrm{~min}$, then $-80{ }^{\circ} \mathrm{C}$ for an overnight and put into liquid nitrogen $\left(-196^{\circ} \mathrm{C}\right)$ for cryopreservation time. Defrost process was 
performed by immersing cryo-vials in a $35^{\circ} \mathrm{C}$ water bath. To remove cryoprotectant, defrosted cell suspensions were centrifuged and supernatant was removed [9]. After that cells were resuspended with 5 $\mathrm{mL}$ of fresh BBM and incubated under $20 \mu \mathrm{mol}$ photons $\mathrm{m}^{-2} \mathrm{~s}^{-1}$ at $22 \pm 2^{\circ} \mathrm{C}$ for 1 week, subsequent to incubation in the dark for $24 \mathrm{~h}$.

\subsection{Viability assay}

After one day thawing, cell viability was measured by the most common staining protocol using fluorescein diacetate (FDA). FDA stock solution was prepared by dissolving of $1 \mathrm{mg}$ flourescein diacetate in $1 \mathrm{~mL}$ of methanol. $50 \mu \mathrm{L}$ of FDA stain solution was added to 1 $\mathrm{mL}$ culture, incubated at $22 \pm 2{ }^{\circ} \mathrm{C}$ for $5 \mathrm{~min}$, and observed by blue-light fluorescence microscopy. Viable cells fluoresce green (positive control) and nonviable cells appeared red or non-color. The images of living cells were taken under 485/535 excitation/emission nm with fluoresce microscope (Olympus BX53, Japan) at $60 \mathrm{X}$ magnification [2].

Cell viability was calculated by the equation (2.2);

$$
\text { Viability }(\%)=\frac{\text { cryopreserved live cell number }}{\text { non-cryopreserved live cell number }} \times 100
$$

\subsection{Measurement of microalgal growth}

Microalgal cell growth was monitored by optical density and cell counting using Neubauer chamber. The optical turbidity was measured at $665 \mathrm{~nm}$ and $750 \mathrm{~nm}$ in spectrophotometer (GE Healthcare Ultrospec 1100 pro, London, UK).

\section{Results and Discussion}

Several factors can potentially influence the success of cryopreservation such as the state and density of the culture, the nature and concentration of the cryoprotectant, the pretreatment with cryoprotectant, the composition and osmolarity of the medium, the cryopreservation time, the rate of cooling, thawing and post-thawing [10]. The most important factors, which effects on algal viability, are the type, concentration and timing of cryoprotective agents, pretreatment with cryoprotectant and duration of cryopreservation [11]. Salas-Leiva and Dupré [12] reported that concentration, temperature and time of exposure are related to the use of cryoprotectant in additionally there are various methods of handling them emphasized in the literature. The cryoprotectant agent as DMSO is used for the treatment of microalgae because it easily permeates cell membranes and its low hydrophilicity [13]. In order to obtain optimum cell viability after cryopreservation, it is necessary to optimize of three factors (cryoprotectant concentrations, incubation time and cryopreservation time) by using response surface methodology (RSM) in analytical optimization [14].

The experimental data were calculated by the DesignExpert software, and the results of each range and level variance analysis were given in Table 3 . The range of variables was selected on test experiences to summarize in databases related to algal cultivation. Response surface methodology (RSM) based on three variables at five level central composite design (CCD) was applied to determine the effect of the cryoprotectant concentration, incubation time and cryopreservation time. As shown in Table 3, 5 different cryoprotectant concentrations; $X_{1}-\%(0,5.07,12.5,19.93,25), 5$ different incubation times; $X_{2}-\min (1,3.84,8,12.96$, $15)$ and 5 different cryopreservation times; $X_{3}-$ day $(7$, $42.07,93.5,144.93,180)$ were tested. Total of 19 experiments were used to optimize the range and levels of selected variables. Besides, the order of treatments was arranged randomly. As seen from Table 3, viability (\%) of the cells are in compatible with the spectrophotometric results and the most vial cells were obtained in the set $1(75 \%)$ and set $11(100 \%)$ for $C$. saccharophila and C. zofingiensis, respectively.

Table 3. Experimental data and levels of the independent variables error in the response surface design.

\begin{tabular}{|c|c|c|c|c|c|c|c|c|c|}
\hline & \multirow{2}{*}{\multicolumn{3}{|c|}{ Independent variables }} & \multirow[t]{2}{*}{ Symbol } & \multicolumn{5}{|c|}{ Levels } \\
\hline & & & & & $-\alpha$ & -1 & $\mathbf{0}$ & +1 & $+\alpha$ \\
\hline & \multicolumn{3}{|c|}{$\begin{array}{c}\text { Cryoprotectant concentration } \\
\text { (DMSO \%) }\end{array}$} & $X_{1}$ & 0 & 5.07 & 12.5 & 19.93 & 25 \\
\hline & \multicolumn{3}{|c|}{ Incubation time (min) } & $X_{2}$ & 1 & 3.84 & 8 & 12.16 & 15 \\
\hline & \multicolumn{3}{|c|}{ Cryopreservation time (day) } & $X_{3}$ & 7 & 42.07 & 93.5 & 144.93 & 180 \\
\hline Run & $\mathrm{X}_{1}(\%)$ & $\mathrm{X}_{2}(\min )$ & $\mathrm{X}_{3}$ (day) & $\begin{array}{l}\text { C. zofingiensis } \\
(665 \mathrm{~nm})\end{array}$ & \multicolumn{2}{|c|}{$\begin{array}{c}\text { Viability } \\
(\%)\end{array}$} & \multicolumn{2}{|c|}{$\begin{array}{c}\text { C. saccharophila } \\
(750 \mathrm{~nm})\end{array}$} & $\begin{array}{c}\text { Viability } \\
(\%)\end{array}$ \\
\hline 1 & 12.5 & 8 & 93.5 & 0.65 & \multicolumn{2}{|c|}{80} & \multicolumn{2}{|c|}{0.105} & 75 \\
\hline 2 & 19.93 & 3.84 & 144.93 & 0.1 & \multicolumn{2}{|c|}{42} & \multicolumn{2}{|c|}{0.029} & 40 \\
\hline 3 & 19.93 & 3.84 & 42.07 & 0.15 & \multicolumn{2}{|c|}{14} & \multicolumn{2}{|c|}{0.033} & 60 \\
\hline 4 & 12.5 & 8 & 93.5 & 0.63 & \multicolumn{2}{|c|}{86} & \multicolumn{2}{|c|}{0.1} & 25 \\
\hline
\end{tabular}




\begin{tabular}{|c|c|c|c|c|c|c|c|}
\hline 5 & 5.06 & 12.16 & 42.07 & 0.08 & 29 & 0.014 & 20 \\
\hline 6 & 5.06 & 3.84 & 144.93 & 0.1 & 15 & 0.029 & 50 \\
\hline 7 & 5.06 & 3.84 & 42.07 & 0.056 & 7 & 0.018 & 25 \\
\hline 8 & 12.5 & 8 & 93.5 & 0.665 & 57 & 0.102 & 25 \\
\hline 9 & 12.5 & 1 & 93.5 & 0.4 & 18 & 0.04 & 20 \\
\hline 10 & 12.5 & 15 & 93.5 & 0.44 & 29 & 0.04 & 25 \\
\hline 11 & 12.5 & 8 & 93.5 & 0.68 & 100 & 0.1 & 25 \\
\hline 12 & 12.5 & 8 & 7 & 0.06 & 13 & 0.02 & 30 \\
\hline 13 & 5.06 & 12.16 & 144.93 & 0.08 & 18 & 0.029 & 35 \\
\hline 14 & 19.93 & 12.16 & 144.93 & 0.138 & 25 & 0.038 & 50 \\
\hline 15 & 12.5 & 8 & 180 & 0.05 & 12 & 0.02 & 20 \\
\hline 16 & 0 & 8 & 93.5 & 0.017 & 12 & 0.029 & 10 \\
\hline 17 & 19.93 & 12.16 & 42.07 & 0.121 & 33 & 0.025 & 20 \\
\hline 18 & 25 & 8 & 93.5 & 0.09 & 11 & 0.035 & 35 \\
\hline 19 & 12.5 & 8 & 93.5 & 0.65 & 11 & 0.103 & 75 \\
\hline
\end{tabular}

Based on the experimental results of $C$. saccharophila (3.1) and C. zoffingiensis (3.2), the following response surface model was explained in the form for the symbol factors $\left(\mathrm{X}_{1} ; \mathrm{X}_{2} ; \mathrm{X}_{3}\right)$ :

$$
\begin{aligned}
& Y=+0.042+1.116 * 10^{-3} * X_{1}-4.222 * 10^{5} * X_{2}+8.364 * 10^{-4} * X_{3}-0.012 * X_{1}^{2}-0.011 * X_{2}^{2}-0.013 * X_{3}^{2} \\
& Y=+0.66+0.023 * X_{1}+5.878 * 10^{-3} * X_{2}-4.260 * 10^{-4} * X_{3}-0.22 * X_{1}^{2}-0.092 * X_{2}^{2}-0.22 * X_{3}^{2}
\end{aligned}
$$

The Analysis of Variance (ANOVA) for the model Equivalent $(2,3)$ of two Chlorella strains observed a good fit between the models and the experimental data. As depicted in Table 4 and 5, the cellular viability of $C$. saccharophila at $750 \mathrm{~nm}$ and C. zofingiensis at $665 \mathrm{~nm}$ had second-degree nominal effect by the three process variables. Tables of ANOVA p-value was measured significance of variable. When both model $p>F$ value of the variable was less than 0.0001 , it represented that the variable had highly significant effects on the response value. The model lack of fit value of 2 implies was significant for $C$. saccharophila, while the F-value of

$$
\text { significant for C. saccharophila, while the F-value of al., } 2012 \text { [16]. }
$$

\begin{tabular}{|c|c|c|c|c|c|}
\hline Source & $* \mathbf{S S}$ & $* \mathbf{D F}$ & *MS & F-value & $\begin{array}{l}\text { p-value } \\
\text { Prob>F }\end{array}$ \\
\hline Model & $4.494 * 10^{-3}$ & 6 & $7.49 * 10^{-4}$ & 305.94 & $<0.0001$ \\
\hline Concentration of DMSO $\left(\boldsymbol{X}_{\boldsymbol{1}}\right)$ & $1.7 * 10^{-5}$ & 1 & $1.7 * 10^{-5}$ & 6.94 & 0.0218 \\
\hline Incubation time $\left(\boldsymbol{X}_{2}\right)$ & $2.434 * 10^{-8}$ & 1 & $2.434 * 10^{-8}$ & $9.942 * 10^{-3}$ & 0.9222 \\
\hline Cryopreservation time $\left(\boldsymbol{X}_{\mathbf{3}}\right)$ & $9.555^{*} 10^{-6}$ & 1 & $9.555^{*} 10^{-6}$ & 3.90 & 0.0717 \\
\hline$X_{1}^{2}$ & $1.867 * 10^{-3}$ & 1 & $1.867 * 10^{-3}$ & 762.51 & $<0.0001$ \\
\hline$X_{2}^{2}$ & $1.541 * 10^{-3}$ & 1 & $1.541 * 10^{-3}$ & 629.63 & $<0.0001$ \\
\hline$X_{3}^{2}$ & $2.349 * 10^{-3}$ & 1 & $2.349 * 10^{-3}$ & 956.62 & $<0.0001$ \\
\hline Residual & $2.938 * 10^{-5}$ & 12 & $2.448 * 10^{-6}$ & & \\
\hline Lack of fit & $2.35 * 10^{-5}$ & 8 & $2.938 * 10^{-6}$ & 2 & 0.2628 \\
\hline Pure error & $5.876^{*} 10^{-6}$ & 4 & $1.469 * 10^{-6}$ & & \\
\hline Corrected total & $4.523 * 10^{-3}$ & 18 & & & \\
\hline Std. Deviation & $1.565 * 10^{-3}$ & & R-Squared & 0.9935 & \\
\hline Mean & 0.016 & & Adj R-Squared & 0.9903 & \\
\hline C.V. $\%$ & 9.55 & & Pred R- Squared & 0.9814 & \\
\hline
\end{tabular}

Table 4. Analysis of variance for response surface design of cryopreserved C. saccharophila on viability at $750 \mathrm{~nm}$. 


\begin{tabular}{|c|c|c|c|}
\hline Press & $8.396 * 10^{-5}$ & Adeq Precision & 40.552 \\
\hline
\end{tabular}

Table 5. Analysis of variance for response surface design of cryopreserved $C$. zofingiensis on viability at $665 \mathrm{~nm}$.

\begin{tabular}{|c|c|c|c|c|c|}
\hline Source & *SS & $* \mathbf{D F}$ & $* \mathbf{M S}$ & F-value & $\begin{array}{l}\text { p-value } \\
\text { Prob>F }\end{array}$ \\
\hline Model & 1.20 & 6 & 0.20 & 230.99 & $<0.0001$ \\
\hline Concentration of DMSO $\left(\boldsymbol{X}_{\mathbf{1}}\right)$ & $7.301 * 10^{-3}$ & 1 & $7.301 * 10^{-3}$ & 8.46 & 0.0131 \\
\hline Incubation time $\left(\boldsymbol{X}_{2}\right)$ & $4.718 * 10^{-4}$ & 1 & $4.718 * 10^{-4}$ & 0.55 & 0.4740 \\
\hline Cryopreservation time $\left(\boldsymbol{X}_{3}\right)$ & $2.478 * 10^{-6}$ & 1 & $2.478 * 10^{-6}$ & $2.870 * 10^{-3}$ & 0.9582 \\
\hline$X_{1}^{2}$ & 0.67 & 1 & 0.67 & 779.44 & $<0.0001$ \\
\hline$X_{2}^{2}$ & 0.12 & 1 & 0.12 & 135.17 & $<0.0001$ \\
\hline$X_{3}^{2}$ & 0.67 & 1 & 0.67 & 775.72 & $<0.0001$ \\
\hline Residual & 0.010 & 12 & $8.634 * 10^{-4}$ & & \\
\hline Lack of fit & $8.961 * 10^{-3}$ & 8 & $1.120 * 10^{-3}$ & 3.20 & 0.1381 \\
\hline Pure error & $1.400 * 10^{-3}$ & 4 & $3.500 * 10^{-4}$ & & \\
\hline Corrected total & 1.21 & 18 & & & \\
\hline Std. Deviation & 0.029 & & R-Squared & 0.9914 & \\
\hline Mean & 0.27 & & Adj R-Squared & 0.9871 & \\
\hline C.V. \% & 10.83 & & Pred R- Squared & 0.9686 & \\
\hline Press & 0.038 & & Adeq Precision & 37.393 & \\
\hline
\end{tabular}

The value of prediction was in good agreement with adjusted $\mathrm{R}^{2}$ emphasizing the significance of this model. Moreover, the closer the $\mathrm{R}^{2}$ value is to 1 , the stronger the design is and the higher it predicts the response [17]. C. saccharophila of regression analysis revealed a coefficient of determination value of 0.9935 and only $0.65 \%$ of the total variations were not explained by this design. Meanwhile, the adjusted correlation coefficient (0.9903) and the predicted correlation coefficient $(0.9814)$ values ratified that the design was good. As an analysis of variance for $C$. zofingiensis is given in Table 5 , the $\mathrm{R}^{2}$, adjusted $\mathrm{R}^{2}$, and predicted $\mathrm{R}^{2}$ values were 0.9914, 0.9871, and 0.9686, respectively, which implied that experimental values could not be enough explained by the design.

These results indicated that the precision and general reliability of the second-order model was quite well and analysis of the response using the model was related to the variation of the factors. The three-dimensional (surface) graphs exhibited the common graphical representation of the regression equation and were shown the optimal values of each dependent variable in Figure 1. Three surface and contour plots were shown to indicate influence of the interaction of cryoprotectant concentration, incubation time and cryopreservation time on cell viability of $C$. saccharophila $(1-\mathrm{a}, \mathrm{b}, \mathrm{c}, \mathrm{d})$ and C. zofingiensis $(2-\mathrm{a}, \mathrm{b}, \mathrm{c}, \mathrm{d})$, respectively. Figure $1-1$ is shown that incubation time and DMSO concentration for $C$. saccharophila followed a concave trend. An increase in the incubation time with increasing the DMSO concentration increased the turbidity gradually up to very specific values and after that point, the viability was decreased dramatically. It was also presented that all variables have significant interactions with each other. As shown Figure 1-2, the response surfaces of $C$. zofingiensis having circular contour lines stated that the interaction between the corresponding variables was less significant than the ones of $C$. saccharophila. Furthermore, a weak effect on the response was observed at the maximum and minimum levels of incubation and cryopreservation times. 

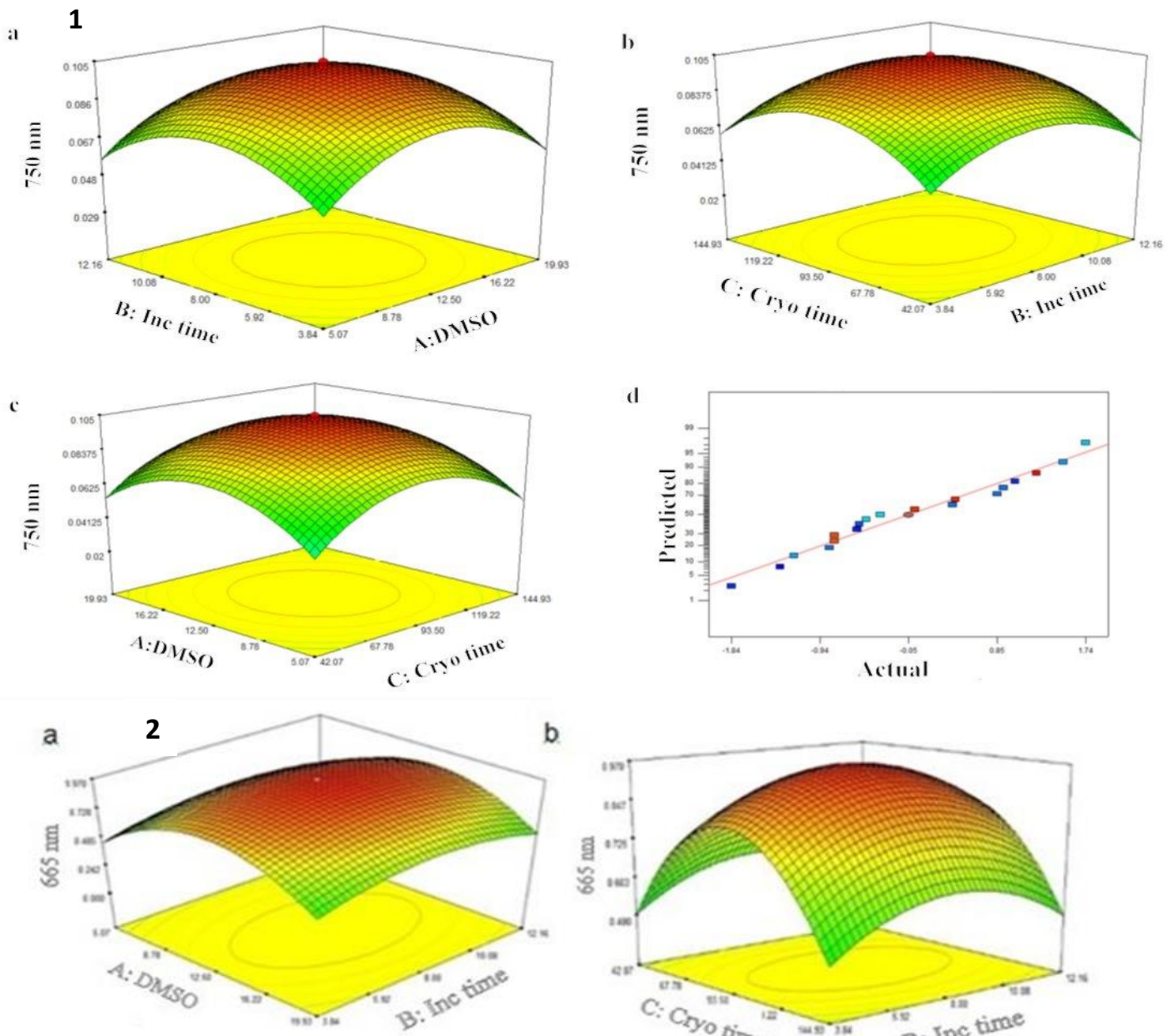

b.
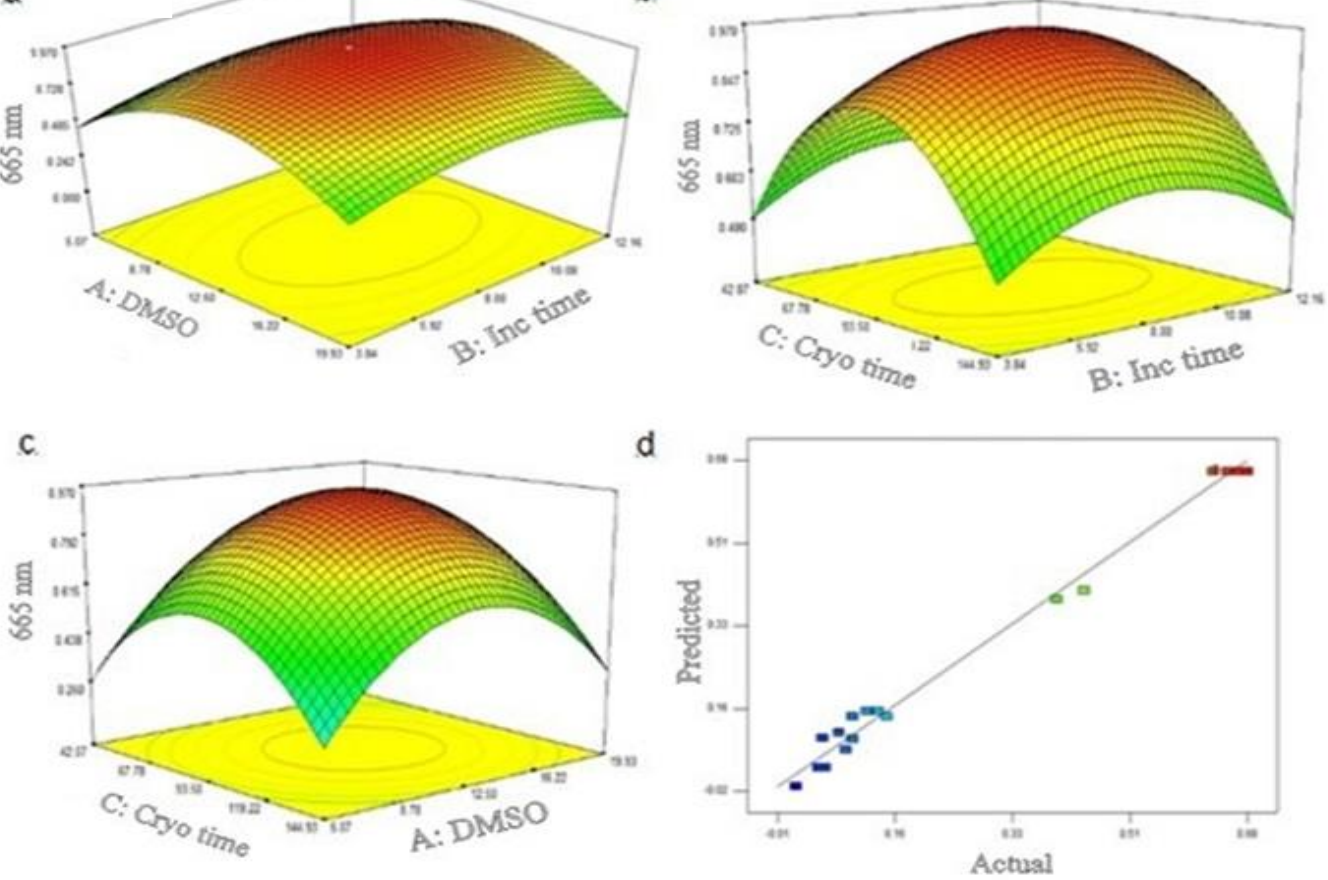

Figure 1. 3D response surface plot of CCD showing the mutual effects of cryoprotectant concentration (DMSO), incubation time (Inc time) and cryopreservation time (Cryo time) on cell viability of $C$. saccharophila (1) and $C$. zofingiensis (2), respectively.

As shown Figure 2, fluorescent dyes fluorescein diacetate (FDA) stains the cytoplasm of live cells, as also visualizing the vacuoles, viable cells fluoresce green and dead and damage cells appeared red or colorless. FDA dye was formed by intracellular hydrolysis, reporting the intact vacuolar and plasma membranes [18]. Figure 2E of $C$. saccharophila and Figure $2 \mathrm{~J}$ of $C$. zofingiensis cells were observed red because chlorophyll autofluorescence was lower in bad physiological state of the cell. Joseph [19] reported that the highest concentration of DMSO where Tetraselmis gracilis, Chaetoceros calcitrans and Chlorella marina cells were viable and found between at $30 \%$ and $40 \%$. Although in DMSO, three strains were viable up to $30 \%$ concentration. Moreover, the effective viability of $C$. marina was also stated at 5\% DMSO. Whereas, in this study and investigation of Canavate and Lubian [20] 
have emphasized that algae could be grown well after cryopreserving in nearly $15 \%$ DMSO. In additional, the study aimed that an evaluation of Chlorella strains incubation time with cryoprotectant at room temperatures had given on loss of viability during the process.
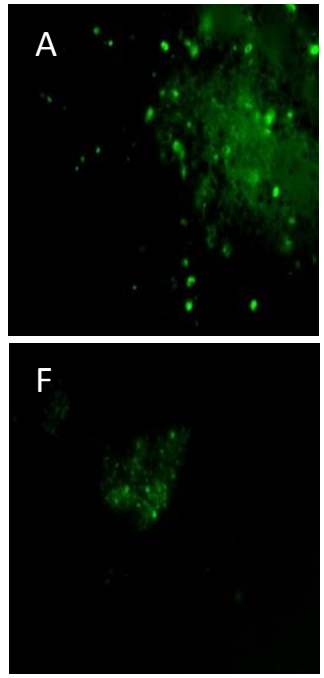
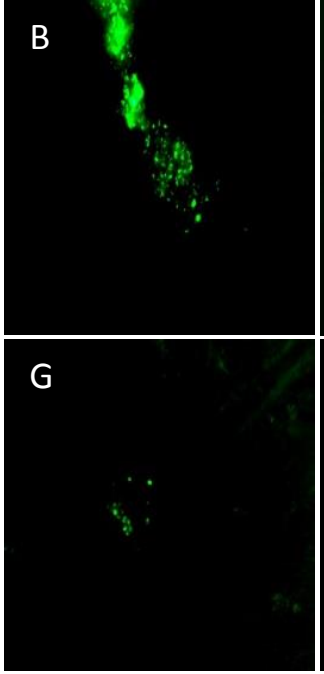
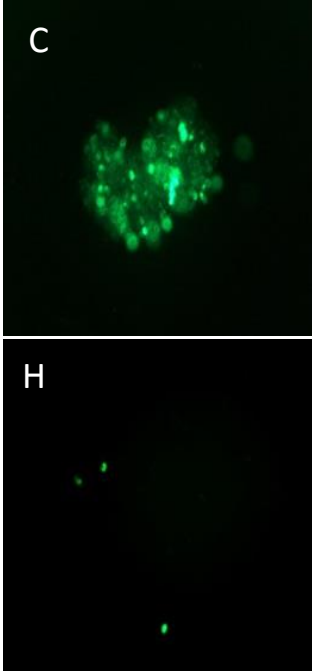
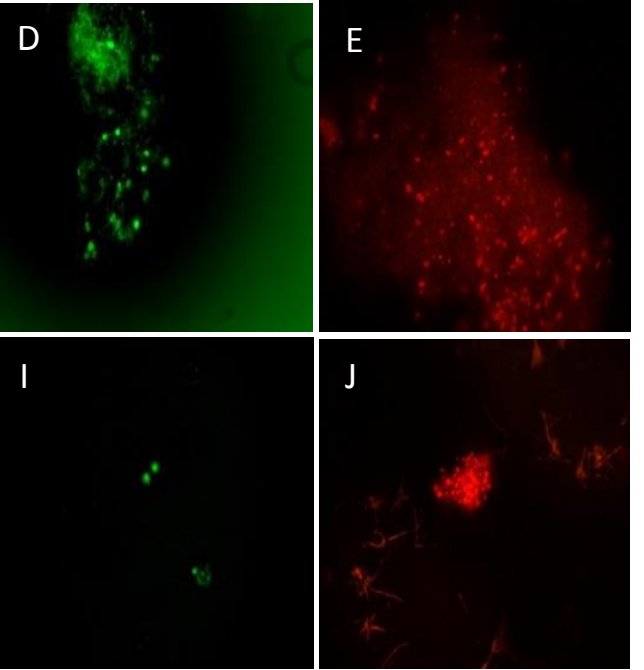

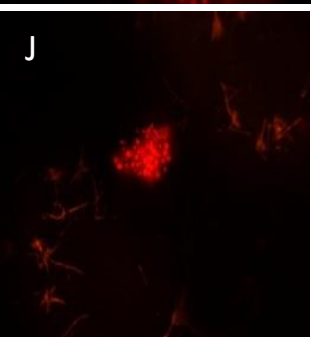

Figure 2. Micrographs of FDA stained microalgae cells. (A-E) C. saccharophila, (F-J) C. zofingiensis. A. $13 \%$ DMSO- day 7, B. 20\% DMSO- day 42, C. 13\% DMSO- day 94, D. 13\% DMSO- day 180, E. 0\% DMSO- day 94, F. 13\% DMSO- day 7, G. 20\% DMSO- day 42, H. 13\% DMSO- day 94, I. 13\% DMSO- day 180, J. 0\% DMSO- day 94.

The validation tests were performed at the optimum conditions to ensure the accuracy of the model in triplicate tests. Optimization of procedure for reply was produced via numerical optimization styles pursuing desirability function.

Optimized results for $C$. saccharophila (at the DMSO concentration of $12.86 \%$ at the incubation time of 7.99 min and at cryopreservation time of 95.17 day) and $C$. zofingiensis (at the DMSO concentration of $12.89 \%$ at the incubation time of $8.14 \mathrm{~min}$ and at cryopreservation time of 93.45 day) were selected and results of the experimental analysis and model predictions were compared. The responses were in good agreement for both strains. For the cultivation of $C$. saccharophila, the experimental response was 0.112 which was closer to the predicted value of 0.102 . Under the optimum conditions of $C$. zofingiensis, the value of prediction maximized response was 0.79 while the experimental result was found to be 0.81 , indicating the accuracy of the model. The verification studies also indicated that designs suitable test results well.

The results of the work showed that cryopreserved microalgae were stored for long term preservation successfully for periods of up to 3 months. The values obtained in this study showed that the selected value range of parameters at the beginning of the study was correct.

\section{Conclusions}

In conclusion, we have demonstrated that the viability of $C$. saccharophila and C. zofingiensis post thaw of

cryopreservation was influenced by parameters such as cryoprotectant concentrations, incubation time and cryopreservation time. In addition, using RSM, the present study was found the cryopreservation conditions for the highest cell viability, seemed to be in keeping with the test values achieved in subsequent validation assay, indicating that RSM may be a useful means for estimating the optimum conditions for test design.

\section{Acknowledgements}

This work has been financially supported by the Scientific and Technological Research Council of Turkey (TUBITAK 113Z202) and Ege University Scientific Research Projects (BAP 14BIL016). The authors thank Çigdem DEMIRKAYA KEKLIK for helping with taking photos.

\section{References}

1. Ravindran, B, Gupta, S.K, Cho, W.M, Kim, J.K, Lee, S.R, Jeong, K.H, Lee, D.J, Choi, H-C, Microalgae Potential and Multiple Roles-Current Progress and Future Prospects-An Overview, Sustainability, 2016, 8(1215), 1-16.

2. Demirel, Z, Demirkaya, C, Imamoglu, E, Dalay, M.C, Diatom cultivation and lipid productivity for non-cryopreserved and cryopreserved cells, Agronomy Research, 2016, 14(4), 12661273 .

3. Nakanishi, K, Deuchi, K, Kuwano, K, Cryopreservation of four valuable strains of microalgae, including viability and characteristics during 15 years of cryostorage, Journal of Applied Phycology, 2012, 24(6), 1381-1385.

4. Kumari, N, Gupta, M.K, Singh, R.K, Open encapsulationvitrification for cryopreservation of algae, Cryobiology, 2016, 73(2), 232-239 
5. Kaur, R, Pramanik, K, Sarangi, S.K, Cryopreservation-induced stress on long-term preserved articular cartilage, ISRN Tissue Engineering, 2013, 2013, 1-10.

6. Day, J.G, Fleck, R.A, Benson, E.E, Cryopreservationrecalcitrance in microalgae: novel approaches to identify and avoid cryo-injury, Journal of Applied Phycology, 2000, 12(3-5), 369-377.

7. Imamoglu, E, Simulation design for microalgal protein optimization, Bioengineered, 2015, 6(6), 342-346.

8. Day, J.G, Brand, J.J, Cryopreservation methods for maintaining microalgal cultures. In: Andersen R.A (ed). Algal culturing techniques, Elsevier Academic Press, London, 2005, pp 165-187.

9. Gaget, V, Chiu, Y.T, Lau, M, Humpage, A.R, From an environmental sample to a long-lasting culture: the steps to better isolate and preserve cyanobacterial strains, Journal of Applied Phycology, 2017, 29(1), 309-321.

10. Poncet, J.M, Cryopreservation of the unicellular marine alga Nannochloropsis oculata, Biotechnology Letters, 2003, 25(23), 2017-2022.

11. Hubalek, Z, Protectants used in the cryopreservation of microorganisms, Cryobiology, 2003, 46(3), 205-229.

12. Salas-Leiva, J.S, Dupré, E, Criopreservación de las microalgas Chaetoceros calcitrans (Paulsen): análisis del efecto de la temperatura de DMSO y régimen de luz durante diferentes períodos de equilibrio, Latin American Journal of Aquatic Research, 2011, 39(2), 271-279.

13. Chen, G, Yue, A, Ruan, Z., Yin, Y, Wang, R, Ren, Y, Zhu, L, Comparison of the effects of different cryoprotectants on stem cells from umbilical cord blood, Stem Cells International, 2016 2016, 1-7.
14. Imamoglu, E, Demirel, Z., Conk Dalay, M, Process optimization and modeling for the cultivation of Nannochloropsis sp. and Tetraselmis striata via response surface methodology, Journal of Phycology, 2015, 51(3), 442-453.

15. Sarrai, A.E, Hanini, S, Merzouk, N.K, Tassalit, D, Szabó, T, Hernádi, K, Nagy, L, Using central composite experimental design to optimize the degradation of tylosin from aqueous solution by photo-fenton reaction, Materials, 2016; 9(428), 1-11.

16. Malakar, J, Nayak, A.K, Goswami S, Use of response surface methodology in the formulation and optimization of bisoprolol fumarate matrix tablets for sustained drug release, ISRN Pharmaceutics, 2012, 2012, 1-10.

17. Imamoglu, E, Demirel, Z, Dalay M.C, Evaluation of culture conditions of locally isolated Dunaliella salina strain EgeMacc024, Biochemical Engineering Journal, 2014, 92, 22-27.

18. Saruyama, N, Sakakura, Y, Asano, T, Nishiuchi, T, Sasamoto, H, Kodama, H, Quantification of metabolic activity of cultured plant cells by vital staining with fluorescein diacetate, Analytical Biochemistry, 2013, 441(1), 58-62.

19. Joseph, I, Panigrahi, A, Chandra, P.K, Tolerance of three marine microalgae to cryoprotectant dimethy sulfoxide, methanol and glycerol, Indian Journal of Geo-Marine Sciences, 2000, 29, 243247.

20. Cañavate, J.P, Lubian, L.M, Tolerance of six marine microalgae to the cryoprotectants dimethyl sulfoxide and methanol, Journal of Phycology, 1994, 30(3), 559-565. 